\title{
EXPRESSION OF AEG-1 AND P53 AND THEIR \\ CLINICOPATHOLOGICAL SIGNIFICANCE IN MALIGNANT LESIONS \\ OF RENAL CELL CARCINOMAS: A MICROARRAY STUDY
}

\author{
Havva Erdem $^{1}$, Murat Oktay ${ }^{1}$, Umran Yildirim ${ }^{2}$, Ali Kemal Uzunlar $^{1}$, M. Ali KayikCi ${ }^{1}$
}

${ }^{1}$ Department of Pathology, Duzce University of Medical Faculty, Duzce, Turkey

2Department of Pathology, Fatih University of Medical Faculty, Ankara, Turkey

\begin{abstract}
The aim of this study was to investigate the relationship of AEG-1 and p53 with the prognostic parameters of renal cell carcinoma (RCC). In this study, 50 paraffin blocks were histopathologically diagnosed at the Department of Pathology of the Medical Hospital of Duzce University, between 2005 and 2011. The cases consisted of 24 clear cell (CC) and 26 non-clear cell (NCC) RCC subtypes as follows: 24 (48\%) clear cell RCC, 12 (24\%) papillary RCC, 4 (8\%) multilocular cystic RCC and 10 (20\%) chromophobe RCC; none had sarcomatoid changes. By immunohistochemical analysis we investigated AEG-1 and p53 expression in carcinomas of the kidney, and by statistical analysis determined their relationship with clinicopathological parameters. Significant relationships were found between increasing tumor diameter and the increase of $\mathrm{p} 53$ ( $\mathrm{p}=0.028$ ). In addition, $\mathrm{p} 53$ was significantly related to renal sinus invasion $(\mathrm{p}=0.05)$ and Fuhrman grade $(\mathrm{p}=0.026)$. There was a significant relationship between increased AEG-1 staining scores and CC and NCC carcinoma subtypes $(\mathrm{p}=0.032)$, tumor capsule invasion $(\mathrm{p}=0.01)$ and lymphovascular invasion $(\mathrm{p}=0.015)$. There was also a significant correlation between tumor size and capsule and lymphovascular invasion $(\mathrm{p}=0.02)$. We concluded that high AEG-1 and p53 expression correlates with the prognostic parameters in RCC patients, and in addition may be associated with tumor progression.
\end{abstract}

Key words: AEG-1, p53, prognostic parameters, renal cell carcinomas.

\section{Introduction}

Renal cell carcinoma (RCC) is the most common malignancy of the adult urinary tract and accounts for approximately $3 \%$ of all adult malignancies [1]. At diagnosis, $25-30 \%$ of patients have metastases and another 20-30\% will develop metastases after nephrectomy although their tumors were localized at diagnosis. Patients with advanced RCC still remain incurable as metastasis is often resistant to hormonal therapies and conventional chemotherapy [2].

AEG-1, also known as metadherin, was first reported in 2002 as a novel late-response gene following HIV-1 infection [3]. AEG-1 was found to serve as a junction protein [4]. Kang et al. first cloned and characterized the full-length human AEG-1 gene. It was found to be located in the cytoplasm, perinuclear regions, nucleolus, and endoplasmic reticulum [5] and to encode a singlepass transmembrane protein with a calculated molecular mass of $64 \mathrm{KDa}$. It contains 12 exons and 11 introns and is $86,082 \mathrm{bp}$ in full length [6-8]. AEG-1 is localized at chromosome 8q22 [7-11]. Subsequently, AEG 1 has been reported to be amplified in a number of malignancies such as hepatocellular carcinoma (HCC), malignant glioma and breast cancer [7-11]. Though it was cloned only nine years ago, this novel gene is known to be a potent mediator in the development of malignancies and is a component of oncogenic signaling path- 
ways. Following its initial identification, AEG-1 was thought to be a potential focus for targeted therapy, based on its multi-faceted role in several significant stages of tumor progression, including transformation, angiogenesis, invasion metastasis, and chemoresistance, and also in the initiation of apoptosis [12].

Renal cancer size affects the tumor suppressor p53, which regulates cellular processes such as DNA repair, apoptosis and cell cycle progression [13]. It is activated and stabilized in response to different types of cellular stress such as hypoxia and oncogenic signaling. DNA damage induces a biological response through the transcriptional regulation of downstream target genes. Responses to p53 activation as well as the molecular mechanisms that dictate the decision of a cell to enter growth arrest or undergo apoptosis are only partially understood [13]. Multiple pathways associated with the pathogenesis and progression of clearcell renal cell carcinoma (CCRCC) have been found, whereas the pathways associated with the pathogenesis of non-clear cell renal carcinomas (NCCRCC) have not been identified to date [14].

The aim of this study was to investigate the expression of AEG-1 and p53 and their clinicopathological significance in CCRCC and NCCRCC.

\section{Material and methods}

This study was carried out using 50 paraffin-embedded kidney tumor samples, which were histopathologically diagnosed at the Department of Pathology of the Medical Hospital of Duzce University between 2005 and 2011.

Areas showing histopathological features of RCC were selected on archival hematoxylin-eosin (HE) slides, and then representative areas of the tumors were marked on the corresponding paraffin blocks for TMA (tissue microarray) construction. Briefly, after the tissue cylinders were taken from the selected regions of the donor paraffin block, they were then punched precisely into a recipient paraffin block using a tissue-arraying instrument. Multiple sections were cut at a thickness of $5 \mu \mathrm{m}$. The slides were stained with the usual HE. All the diagnoses were performed by two pathologists following the World Health Organization Classification of Tumors criteria.

\section{Immunohistochemistry}

Among the hematoxylin-eosin-stained slides, one suitable paraffin block was chosen. For p53, Zymed brand BP53-12 clone from Lab Inc. (San Francisco, CA, U.S.A.) was used, diluted at a ratio of $1: 80$. For AEG-1, Genetex brand 2F11C3 clone was used, diluted at a ratio of $1: 200$. Staining procedures were carried out manually. Initially, 5-micron-thick sections were obtained from the tissues and kept in an incubator at $48^{\circ} \mathrm{C}$ overnight, according to the $\mathrm{ABC}$ technique. After being kept in xylene for $5 \mathrm{~min}$, each section was transferred into absolute alcohol (ethyl alcohol) for $15 \mathrm{~min}$, then rinsed in bidistilled water, after which a target retrieval procedure was conducted in a microwave oven for $3 \times 5 \mathrm{~min}$ in a citrate buffer $(\mathrm{pH}=9)$. After immersing it in $3 \%$ hydrogen peroxide for $15 \mathrm{~min}$, the section was washed with phosphate buffer solution (PBS). Then, the primary antibody (p53) was added with a dropper and it was incubated for $40 \mathrm{~min}$. The sample was then washed in PBS and kept for 15 min after secondary antibody drops were added. After that, it was washed with PBS again and incubated for 5-6 min after the addition of Dab Chromogen. After being washed in tap water, the section was counterstained with hematoxylin and sealed with a water-based solution.

Two observers reviewed and scored the degree of immunostaining independently, based on both the proportion of positively stained tumor cells and the intensity of staining. The proportion of tumor cells was scored as follows: 0 (no positive tumor cells), 1 ( $<10 \%$ positive tumor cells), 2 (10-50\% positive tumor cells), and 3 ( $>50 \%$ positive tumor cells). The intensity of staining was graded according to the following criteria: 0 (no staining); 1 (weak staining = light yellow), 2 (moderate staining = yellow brown), and 3 (strong staining $=$ brown). The staining index was calculated as the staining intensity score proportion of the positive tumor cells. Using this method of assessment, we evaluated the expression of AEG-1 in normal tubular epithelium and malignant lesions by determining the staining index, scored as $0,1,2,3,4,6$, and 9. The staining index score of $\geq 4$ was used to define tumors as high AEG-1 expression and $\leq 3$ as low expression of AEG-1 [15] (Fig. 1). Moderate to strong cytoplasmic staining of AEG-1 protein was observed in tumor cells in RCC tissues. The scoring of p53 was as follows: 0 (no positive tumor cells), 1 ( $<10 \%$ positive tumor cells), 2 (10-50\% positive tumor cells), and 3 ( $>50 \%$ positive tumor cells), as seen in Fig. 2.

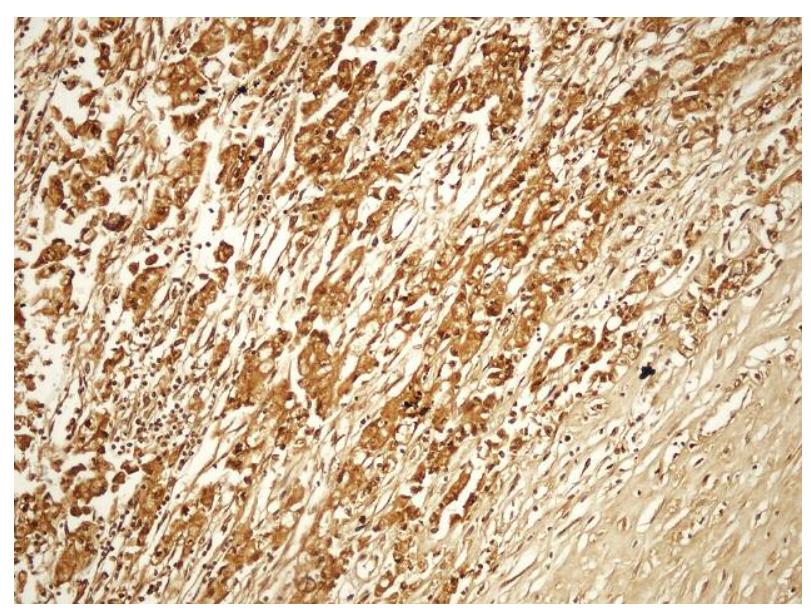

Fig. 1. AEG-1 expression in tumor tissue (magnification $200 \times$, grade 1 ) 


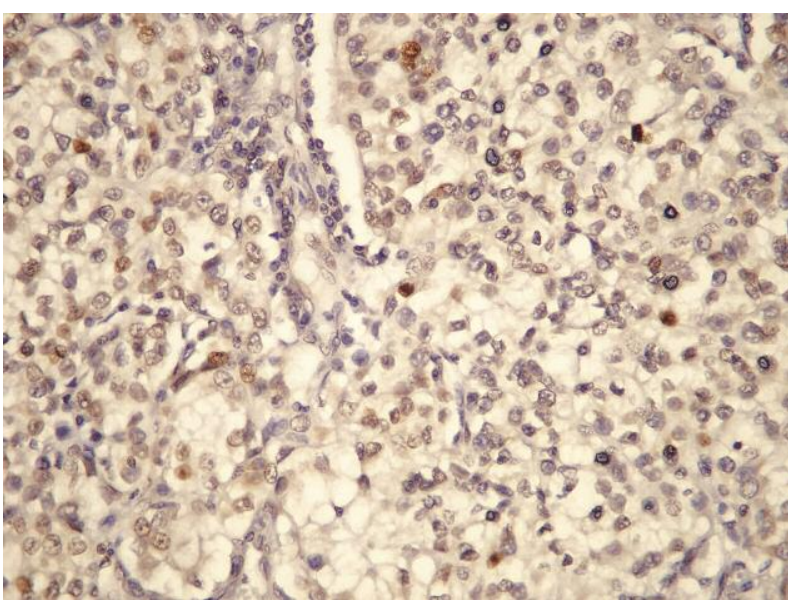

Fig. 2. P53 expression in tumor tissue (magnification $400 \times$, grade 1 )

\section{Statistical analysis}

Descriptive statistics were computed as mean \pm SD or count and percent frequency. The Spearman correlation analysis or likelihood chi-square test analysis (whichever was deemed appropriate in each case) was used to evaluate the relationship between prognostic factors and the degree of staining, and the relation of the staining results to each other. The level of significance was determined to be 0.05 . Statistical analyses were performed using PASW (ver. 18).

\section{Results}

Twelve (24\%) of the patients were female and 38 (76\%) were male. The age distribution of the cases ranged from 27 (the lowest) to 96 (the highest), with the median age at 62.5. There were 11 patients $(22 \%)$ under the age of 50, 16 patients (32\%) aged 50-64, 13 patients (26\%) aged $65-74$ and 10 patients (20\%) over the age of 74 .
The subtypes of the cases included $24(48 \%)$ clear cell RCC, 12 (24\%) papillary RCC, 4 (8\%) multilocular cystic RCC, and 10 (20\%) chromophobe RCC. None of the cases had sarcomatoid changes. There were 24 CCRCC and 26 NCCRCC cases in total.

The tumor stage (pT) distribution was as follows: 23 patients $(46 \%)$ pTla, with tumors $4 \mathrm{~cm}$ or less in diameter, 15 patients $(30 \%) \mathrm{pT} 1 \mathrm{~b}$, with tumors more than $4 \mathrm{~cm}, 1$ patient (2\%) pT2 and 11 patients (22\%) pT3a, with tumors of more than $7 \mathrm{~cm}$. Lymphovascular invasion was positive in 34 cases (78\%) and negative in 16 cases $(32 \%)$. Capsule invasion was also positive in 34 cases and negative in 16 cases. In 6 cases (12\%), both lymphovascular and capsular invasion were determined. Renal sinus invasion was negative in 39 cases $(78 \%)$ and positive in 11 cases $(22 \%)$. The Fuhrman nuclear grade distribution (for clear cell RCC and papillary RCC) was as follows: $15(30 \%)$ grade 1, $18(36 \%)$ grade 2, $3(6 \%)$ grade $3,0(0 \%)$ grade 4 . Neither the renal vein nor the vena cava was observed beyond Gerota's fascia.

\section{Evaluation of immunohistochemical results with prognostic parameters}

There was a significant relationship between the increased diameter and the increase of $\mathrm{p} 53(\mathrm{p}=0.028)$, as shown in Table I. Moreover, p53 was significantly related to renal sinus invasion and the Fuhrman grade (Table I). There was a significant relationship between increased AEG-1 staining scores and clear and non-clear carcinoma subtypes $(\mathrm{p}=0.032)$ as well as between increased AEG-1 staining scores and tumor capsule invasion $(\mathrm{p}=0.01)$ and lymphovascular invasion ( $\mathrm{p}=0.015)$, as seen in Table II. A significant positive correlation was found between tumor size and capsule and lymphovascular invasion $(\mathrm{p}=0.02)$, as shown in Table III. There were no significant relationships between other prognostic parameters.

Table I. Relationship between prognostic parameters [capsule invasion, size and lymphovascular invasion (LVI)]

\begin{tabular}{lcccc}
\hline DiAmETER & & $\leq 7 \mathrm{CM}$ & $>7 \mathrm{CM}$ & $\mathbf{P}$ \\
\hline Capsule & negative $(\mathrm{n})$ & $34(77.3 \%)$ & $10(22.7 \%)$ & \\
invasion & positive $(\mathrm{n})$ & $1(16.7 \%)$ & $83.3 \%$ & 0.02 \\
& negative $(\mathrm{n})$ & $34(77.3 \%)$ & $10(22.7 \%)$ & \\
& positive $(\mathrm{n})$ & $1(16.7 \%)$ & $5(83.3 \%)$ & \\
\hline
\end{tabular}

Table II. Relationship between increased AEG staining score and carcinoma subtypes (clear and non-clear) and capsule invasion $(\mathrm{CI})$

\begin{tabular}{lcccc}
\hline & AEG-1 & LOw & HIGH & \\
\cline { 3 - 4 } & & & $\mathbf{N}$ & P \\
\hline \multirow{2}{*}{ Subtype } & CCRCC & $19(79.2 \%)$ & $5(20.8 \%)$ & \multirow{2}{*}{0.032} \\
\cline { 2 - 4 } & NCCRCC & $13(50.0 \%)$ & $13(50.0 \%)$ & \\
\hline CI & negative & $31(100.0 \%)$ & $0(0.0 \%)$ & 0.01 \\
\cline { 2 - 4 } & positive & $13(68.4 \%)$ & $6(31.6 \%)$ & \\
\hline
\end{tabular}


Table III. Relationship between P53 and prognostic parameters (diameter, Fuhrman grade, renal sinus invasion)

\begin{tabular}{|c|c|c|c|c|c|c|}
\hline & \multicolumn{3}{|c|}{ P53 } & & & \multirow[t]{2}{*}{$\mathbf{P}$} \\
\hline & GRADE & $0(\mathrm{~N})$ & $1(\mathrm{~N})$ & $2(\mathrm{~N})$ & $3(\mathrm{~N})$ & \\
\hline \multirow[t]{2}{*}{ Size } & $\leq 7 \mathrm{~cm}$ & $5(100 \%)$ & $10(52.6 \%)$ & $13(92.9 \%)$ & $7(58.3 \%)$ & \multirow[t]{2}{*}{0.028} \\
\hline & $>7 \mathrm{~cm}$ & 0 & $9(47.4 \%)$ & $1(7.1 \%)$ & $5(41.7 \%)$ & \\
\hline \multirow[t]{2}{*}{ Renal sinus } & negative & $5(12.8 \%)$ & $14(35.9 \%)$ & $13(33.3 \%)$ & $7(17.9 \%)$ & \multirow[t]{2}{*}{0.05} \\
\hline & positive & 0 & $5(45.5 \%)$ & $1(9.1 \%)$ & $5(45.5 \%)$ & \\
\hline \multirow[t]{3}{*}{ Fuhrman grade } & 1 & $1(7.0 \%)$ & $2(14.0 \%)$ & $6(42 \%)$ & $5(35 \%)$ & \multirow[t]{3}{*}{0.026} \\
\hline & 2 & $2(25.0 \%)$ & $5(62.5 \%)$ & 0 & $1(12.5 \%)$ & \\
\hline & 3 & 0 & $2(50.0 \%)$ & $1(25.0 \%)$ & $1(25.0 \%)$ & \\
\hline
\end{tabular}

\section{Discussion}

In recent years, numerous studies have demonstrated that AEG-1 is upregulated and correlated with the progression and prognosis of the patients [16-18]. Overexpression of AEG-1 has been significantly correlated with the clinical stage and various tumor grading parameters $[19,20]$. Chen $e t$ al. found a relationship between AEG-1 staining and the clinical characteristics of patients [15]. AEG-1 has been demonstrated to play a role in several significant stages of tumor progression [15-17]. The tumorigenic potential of AEG-1 is supported by two observations of elevated expression in subsets of cancer cell lines [6]. The present study found a relationship between AEG-1 staining and clinical parameters including subtypes (clear cell and non-clear cell) and tumor capsule and lymphovascular invasion. There was more staining with NCCRCC than CCRCC and higher staining in positive capsule invasion RCC. However, low-grade AEG-1 produced more staining in negative capsule invasion than in positive capsule invasion RCC.

Lee et al. reported that younger patients were more likely to have non-clear cell RCC with higher disease recurrence and lower overall survival $\{21]$. The present study found high-stain AEG-1 (cytoplasmic) in nonclear cell RCC.

Multivariate analysis indicated that AEG-1 expression alone is a poor prognostic indicator for different carcinomas. Furthermore, Yu et al. suggested that AEG-1 expression is independently a poor prognostic indicator for esophageal squamous cell carcinoma patients [17].

Liao et al. reported that moderate to strong cytoplasmic staining of AEG-1 protein was observed in tumor cells of primary salivary gland carcinoma tissue. By contrast, weak or negative signals were observed in normal control tissue. They found that AEG-1 expression was strongly correlated with tumor stage and TNM classification. However, their analyses did not show significant associations between AEG-1 expression and other clinical features, including age, gender, histological type and history of drinking and smoking [22].

In the present study, AEG-1 has shown cytoplasmic staining in tumors. In addition, AEG-1 expression was found to be associated with tumor capsule invasion RCC $(p=0.01)$, lymphovascular invasion RCC $(p=0.015)$ and with the NCCRCC subtype $(\mathrm{p}=0.032)$.

AEG-1 has also been associated with an increased Fuhrman grade and shorter patient survival [19, 20]. Chen et al. found that AEG-1 expression was significantly correlated with the Fuhrman nuclear grade [15]. Unfortunately, we did not find any correlation with the Fuhrman nuclear grade.

Our results suggest that AEG-1 could be a valuable biomarker for the prediction of NCCRCC prognosis.

AKT can inactivate $\mathrm{p} 53$, contributing to centrosome hyperamplification and chromosome instability in cancer [23-25]. Luo et al. and Carroll et al. have provided an in-depth focus on the role of the PI3K/AKT pathway in cell proliferation and survival $[26,27]$.

Although the ability of p 53 to induce apoptosis is known, its prognostic significance for RCC remains controversial [24-29]. Sejima et al. have shown the alteration of apoptotic-regulatory molecule expression during carcinogenesis by comparing tumoral expression with that of normal tissue. They have shown the alteration of apoptotic-regulatory molecule expression during tumor progression by evaluating the relationships of expression with pathological and clinical characteristics of RCC [28].

Baytekin et al. did not observe a correlation between p53 and histopathological type; however, an inverse correlation was found between $\mathrm{p} 53$ expression and tumor stage $(p=0.014)$ and the Fuhrman nuclear grade $(p=0.04)$ in RCC [29].

Cho et al. showed that p53 expression strongly correlated with the TNM stage and the survival rate of patients correlated with the $\mathrm{p} 53$ expression. The expression of $\mathrm{p} 53$ was independent of prognostic factors for cancer-specific survival. They showed that the increased expression of $\mathrm{p} 53$ was associated with metastasis and a worse prognosis in conventional RCC [30]. In the present study, we found that p53 expression was significantly correlated with the tumor size, renal sinus invasion and Fuhrman nuclear grade. Our results suggest that $\mathrm{p} 53$ could be a valuable biomarker for the prediction of poor prognosis. 
Hodorova et al. found that $\mathrm{p} 53$ expression was 4 to 5 times higher $(30.8 \%)$ in other types of RCC than in the clear-cell type of RCC (6.9\%) [31]. The present study did not find any relationship between p53 and tumor subtypes.

\section{Conclusions}

Our results support the idea that AEG-1 and p53 play a role in the progression and carcinogenesis of RCC. Statistical analysis suggests that it is possible to use AEG-1 and p53 as clinically relevant indicators for disease progression. In conclusion, as a result of the clarification of the relationship of $\mathrm{p} 53$ and AEG-1 with RCC, these markers will be useful for the development of new treatments.

The authors declare no conflict of interest.

\section{References}

1. Nelson EC, Evans CP, Lara PN Jr. Renal cell carcinoma: current status and emerging therapies. Cancer Treat Rev 2007; 33: 299-313.

2. Curti BD. Renal cell carcinoma. JAMA 2004; 292: 97-100.

3. Su ZZ, Kang DC, Chen YL. Identification and cloning of human astrocyte genes displaying elevated expression after infection with HIV-1 or exposure to HIV-1 envelope glycoprotein by rapid subtraction hybridization, RaSH. Oncogene 2002; 21: 3592-3602.

4. Britt DE, Yang DF, Yang DQ, et al. Identification of a novel protein, LYRIC, localized to tight junctions of polarized epithelial cells. Exp Cell Res 2004; 300: 134-148.

5. Sutherland HG, Lam YW, Briers S, et al. 3D3/lyric: a novel transmembrane protein of the endoplasmic reticulum and nuclear envelope, Which is also present in the nucleolus. Exp Cell Res 2004; 294: 94-105.

6. Kang DC, Su ZZ, Sarkar D, et al. Cloning and characterization of HIV-1-inducible astrocyte elevated gene-1, AEG-1. Gene 2005; 353: 8-15.

7. Kim DH, Mohapatra G, Bollen A, et al. Chromosomal abnormalities in glioblastoma multiforme tumors and glioma cell lines detected by comparative genomic hybridization. Int J Cancer 1995; 60: 812-819

8. Poon TC, Wong N, Lai PB, et al. A tumor progression model for hepatocellular carcinoma: bioinformatic analysis of genomic data. Gastroenterology 2006; 131: 1262-1270.

9. Wang Y, Klijn JG, Zhang Y, et al. Geneexpression profiles to predict distant metastasis of lymph-node-negative primary breast cancer. Lancet 2005; 365: 671-679.

10. van 't Veer LJ, Dai H, van de Vijver MJ, et al. Gene expression profiling predicts clinical outcome of breast cancer. Nature 2002; 415: 530-536.

11. van de Vijver MJ, He YD, van't Veer LJ, et al. A gene expression signature as a predictor of survival in breast cancer. N Engl J Med 2002; 347: 1999-2009.

12. Liu X, Zhang N, Li X, et al. Identification of novel variants of metadherin in breast cancer. PLoS One 2011, 6 :e17582.

13. Vousden $\mathrm{KH}, \mathrm{Lu} \mathrm{X}$. Live or let die: the cell's response to p53. Nat Rev Cancer 2002; 2: 594-604.
14. Chowdhury S, Matrana MR, Tsang C, et al. Systemic therapy for metastatic non-clear-cell renal cell carcinoma: recent progress and future directions. Hematol Oncol Clin North Am 2011; 25: 853-869.

15. Chen W, Ke Z, Shi H, et al. Overexpression of AEG-1 in renal cell carcinoma and its correlation with tumor nuclear grade and progression. Neoplasma 2010; 57: 522-529.

16. Li J, Zhang N, Song LB, et al. Astrocyte elevated gene- 1 is a novel prognostic marker for breast cancer progression and overall patient survival. Clin Cancer Res 2008; 14: 3319-3326.

17. Yu C, Chen $\mathrm{K}$, Zheng $\mathrm{H}$, et al. Overexpression of astrocyte elevated gene-1 (AEG-1) is associated with esophageal squamous cell carcinoma (ESCC) progression and pathogenesis. Carcinogenesis 2009; 30: 894-901.

18. Song L, Li W, Zhang H, et al. Over-expression of AEG-1 significantly associates with tumour aggressiveness and poor prognosis in human nonsmall cell lung cancer. J Pathol 2009; 219: 317-326.

19. Fuhrman SA, Lasky LC, Limas C. Prognostic significance of morphologic parameters in renal cell carcinoma. Am J Surg Pathol 1982; 6: 655-663.

20. Thirkettle HJ, Girling J, Warren AY, et al. LYRIC/AEG-1 is targeted to different subcellular compartments by ubiquitinylation and intrinsic nuclear localization signals. Clin Cancer Res 2009; 15: 3003-3013.

21. Lee LS, Yuen JS, Sim HG. Renal cell carcinoma in young patients is associated with poorer prognosis. Ann Acad Med Singapore 2011; 40: 401-406.

22. Liao WT, Guo L, Zhong Y, et al. Astrocyte elevated gene-1 (AEG-1) is a marker for aggressive salivary gland carcinoma. J Transl Med 2011; 9: 205.

23. Sherr CJ, Weber JD. The ARF/p53 pathway. Curr Opin Genet Dev 2000; 10: 94-99.

24. Girgin C, Tarhan H, Hekimgil M, et al. p53 mutations and other prognostic factors of renal cell carcinoma. Urol Int 2001; 66: 78-83.

25. Uchida T, Gao JP, Wang C, et al. Clinical significance of $\mathrm{p} 53$, $\mathrm{mdm} 2$, and bcl-2 proteins in renal cell carcinoma. Urology 2002; 59: 615-620.

26. Luo J, Manning BD, Cantley LC. Targeting the PI3K-Akt pathway in human cancer: rationale and promise. Cancer Cell 2003; 4: 257-262.

27. Carroll PE, Okuda M, Horn HF, et al. Centrosome hyperamplification in human cancer: chromosome instability induced by p53mutation and/or Mdm2 overexpression. Oncogene 1999; 8: 1935-1944.

28. Sejima T, Isoyama T, Miyagawa I. Alteration of apoptotic regulatory molecules expression during carcinogenesis and tumor progression of renal cell carcinoma. Int J Urol 2003; 10: 476484 .

29. Baytekin F, Tuna B, Mungan U, et al. Significance of P-glycoprotein, $\mathrm{p} 53$, and survivin expression in renal cell carcinoma. Urol Oncol 2011; 29: 502-507.

30. Cho DS, Joo HJ, Oh DK, et al. Cyclooxygenase- 2 and p 53 expression as prognostic indicators in conventional renal cell carcinoma. Yonsei Med J 2005; 46: 133-140.

31. Hodorova I, Solar P, Mihalik J, et al. Investigation of tumour supressor protein $\mathrm{p} 53$ in renal cell carcinoma patients from Slovakia. Biomed Pap Med Fac Univ Palacky Olomouc Czech Repub 2012 Jun 1. doi: 10.5507/bp.2012.035.

\section{Address for correspondence}

Havva Erdem

Department of Pathology

Duzce University of Medical Faculty

Duzce, Turkey

e-mail: drhavvaerdem@hotmail.com 